\title{
Measuring Steady-state Visual Evoked Potentials from Non-hair-bearing Areas
}

Abstract No:

7443

Authors:

Yu-Te Wang $^{1}$, Yijun Wang ${ }^{1}$, Tzyy-Ping Jung ${ }^{1}$

Institutions:

${ }^{1}$ University of California, San Diego, California, United States

Introduction:

Steady-state visual evoke potential (SSVEP) is the electrical response of the brain to flickering visual stimuli. Due to its advantages such as little user training and high information transfer rate (ITR), the SSVEP has been widely used to implement brain-computer interface (BCI) systems (Wang et al., 2008). In order to achieve a high signal-to-noise ratio (SNR), intuitively, electrodes need to be placed as close as possible to the visual cortex. However, for EEG recording over the haircovered occipital areas, skin and electrode preparation is one of the practical problems that hinder real-life BCI applications. If the SSVEP signals can be measured on non-hair locations on the scalp, the practicality of a BCI system can be significantly improved, especially when dry EEG sensors are applied (Chi et al., 2011; Lin et al., 2008).

The topography of SSVEP often shows a widespread scalp distribution. Therefore, we hypothesize that the non-hair areas at the back of the head might be used for measuring SSVEPs. This study aims to provide a quantitative analysis of SSVEP signals recorded from non-hair locations.

\section{Methods:}

We recorded 256-channel EEG signals from an experienced user of an SSVEP-based BCI. Besides the typical brain areas (i.e., the international 10-20 system), electrode placements also covered the face and the neck areas (Figure 1 ). The subject was seated in a comfortable armed chair about $1 \mathrm{~m}$ from a $5 \mathrm{~cm} \times 5 \mathrm{~cm}$ box rendered at the center of a screen. The stimulus was coded at five frequencies from 9 to $13 \mathrm{~Hz}$ (Wang et al., 2010). For each frequency, two one-minute-long EEG data were recorded, and partitioned into 30 4-second-long trials. Fast Fourier transform (FFT) was applied to the averaged waveforms of the segmented data for estimating the power spectral density (PSD).

This study used SNR to evaluate the efficiency of SSVEP detection. SNR was defined as the ratio of the amplitude of SSVEP (at the stimulating frequency) to the mean amplitude of the background noise (within the frequency band of $8-15 \mathrm{~Hz}$ ). Specifically, this study compared the SNR of four regions including the occipital region, the area behind the ear, the neck area, and the face area. One electrode was selected from each region to represent the SSVEP of the region (Figure 1).

To further test the practicality of the proposed method in BCI applications, we conducted an on-line BCI test on the subject using a four-channel cell-phone-based BCI system (Wang et al., 2011). The four electrodes were placed over the non-hair areas behind the ears (two electrodes on each side) closest to the visual cortex. The reference electrode was placed on the forehead. The Canonical Correlation Analysis (CCA) method (Bin et al., 2009) was applied to multi-channel EEG data for frequency detection. In each of three bouts, the subject was asked to input a 10-digit phone number using a virtual keypad. The accuracy and ITR were used to evaluate the performance of the BCI.

\section{Results:}

Figure 1 shows the normalized PSD of the averaged 10-Hz SSVEP signals. An SNR of 27.28, 16.2, 10.28 and 7.56 was obtained for the occipital area, the behind-ear area, the face area, and the neck area respectively. Figure 2 shows the SNR of the $10-\mathrm{Hz}$ SSVEPs across the whole head. The nonhair areas show reliable SSVEP response although the amplitude was much lower than the occipital region. In the online BCI test, the subject achieved an ITR of $22.1 \mathrm{bits} / \mathrm{min}$ (with an accuracy of $83.33 \%$ ), which was comparable to other BCI systems. 


\section{Conclusions:}

The results of this study showed that EEG recordings from non-hair areas could reliably measure SSVEPs. Thus, the areas behind the ears can be a good alternative to the hair-covered occipital lobe for realizing an SSVEP-based BCI. The proposed approach can significantly improve the practicality of BCI systems in real-life environments.

\section{Motor Behavior:}

Brain Machine Interface
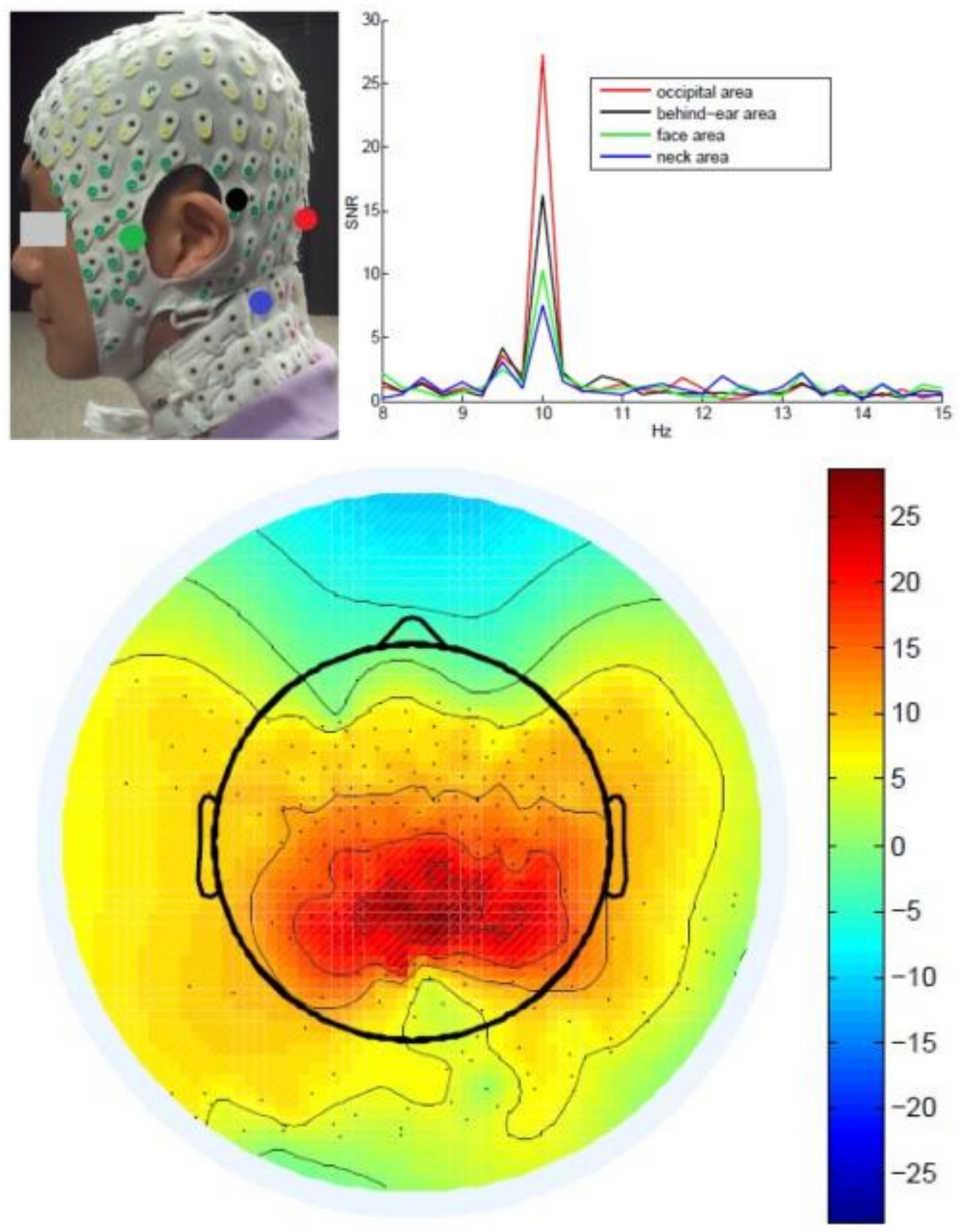

\section{Abstract Information}

\section{References}


Wang, Y., Gao, X., Hong, B., Jia, C., Gao, S. (2008), 'Brain-computer interfaces based on visual evoked potentials: feasibility of practical system designs', IEEE Engineering in Medicine and Biology Magazine, vol. 27, no. 5, pp. 64-71.

Chi, Y. M., Wang, Y. T., Wang, Y., Maier, C., Jung, T. P., Cauwenberghs, G. (in press), 'Dry and non-contact EEG sensors for mobile brain-computer interfaces', IEEE Transactions on Neural Systems and Rehabilitation Engineering.

Lin, C. T., Ko, L. W., Chiou, J. C., Duann, J. R., Chiu, T. W., Huang, R. S., Liang, S. F., Jung, T. P. (2008), 'A noninvasive prosthetic platform using mobile and wireless EEG', Proceedings of the IEEE, vol. 96, no. 7, pp. 1167-83.

Wang, Y., Wang, Y. T., Jung, T. P. (2010), 'Visual stimulus design for high-rate SSVEP BCI', Electronics Letters, vol. 46, no. 15, pp. 1057-1058.

Wang, Y. T., Wang, Y., Jung, T. P. (2010), 'A cell-phone based brain computer interface for communication in daily life', Journal of Neural Engineering, vol. 8, no. 2, pp. 1-5.

Bin, G., Gao, X., Yan, Z., Hong, B., Gao, S. (2009), 'An online multi-channel SSVEP-based braincomputer interface using a canonical correlation analysis method', Journal of Neural Engineering, vol. 6, no. 4, pp. 046002. 